\title{
Time gated phase-correlation distributed Brillouin fibre sensor
}

\author{
Andrey Denisov*, Marcelo A. Soto, Luc Thévenaz \\ Ecole Polytechnique Fédérale de Lausanne, Institute of Electrical Engineering, \\ Group for Fibre Optics SCI-STI-LT Station 11, CH-1015 Lausanne, Switzerland \\ *E-mail: andrey.denisov@epfl.ch
}

\begin{abstract}
A random access distributed Brillouin fibre sensor is presented, based on phase modulation using a pseudo-random bit sequence (PRBS) together with time gating. The standard phase-correlation technique is known to show a noise level increasing linearly with the number of measured points due to weak gratings generated randomly along the whole sensing fibre. Here we show how intensity modulated pump and time gated detection significantly improve the signal-tonoise ratio (SNR) of the system with no impact on the spatial resolution. A measurement with $1.1 \mathrm{~cm}$ spatial resolution over $3.3 \mathrm{~km}$ is demonstrated, representing 300'000 equivalent points. The limitations of the proposed technique are discussed through the paper.
\end{abstract}

Keywords: Fibre optics, optical fibre sensors, nonlinear fibre optics, Brillouin scattering, distributed fibre sensor.

\section{INTRODUCTION}

Brillouin fibre sensors have been used for more than twenty years for temperature and strain monitoring. One of the goals for the research in this field has steadily been to increase the number of resolved points either by extending the length of the sensing fibre or by improving the spatial resolution.

The spatial resolution of sensors based on Brillouin optical time-domain analysis/reflectometry (BOTDA/BOTDR) is primarily limited to 1 meter due to the acoustic decay time of $10 \mathrm{~ns}$. Various techniques have been proposed to overcome this limitation: Brillouin echoes were proved to provide $5 \mathrm{~cm}$ spatial resolution over $5 \mathrm{~km}^{1}$ and recently $2 \mathrm{~cm}$ resolution over $2 \mathrm{~km}$ distance has been demonstrated using differential pulse-widths ${ }^{2}$. But since these techniques work in time domain they require high-speed equipment for pulse generation and detection (e.g. $10 \mathrm{GHz}$ for $1 \mathrm{~cm}$ spatial resolution).

Other techniques avoid using pulses and rather localize Brillouin interaction using correlation based schemes. Brillouin optical correlation-domain analysis (BOCDA) and a phase-correlation technique based on modulating the phases of the interacting waves by a PRBS both have proved to resolve sub-centimetre spatial features. However the number of resolved points for BOCDA is limited to 570 by the method itself (using multiple modulation schemes can increase this number to $2^{\prime} 800$ points $^{3}$ ). For the phase-correlation technique $20^{\prime} 000$ resolved points have been demonstrated ${ }^{4}$, but the problem of this technique is that the noise level scales linearly with the number of points. It has been estimated that for more than 100 '000 points it turns out to be practically impossible to distinguish the real signal from the noise ${ }^{4}$.

In this paper we demonstrate how intensity-modulated pump and time-gated detection applied to the phase-correlation technique significantly reduce the noise level while maintaining the spatial resolution. A $1.1 \mathrm{~cm}$ hotspot is successfully detected at the end of a $3.3 \mathrm{~km}$ long fibre thus increasing the number of resolved points by an order of magnitude in comparison to phase-correlation technique with continuous waves.

By reaching 300'000 resolved points the here presented technique has achieved the largest number of distinct measured points that was ever realized in a distributed Brillouin fibre sensor.

\section{TIME GATED PHASE-CODING TECHNIQUE.}

The phase-correlation technique ${ }^{4}$ is based on using a high-speed PRBS to modulate phases of both pump and probe waves applying either 0 or $\pi$ phase shift. It leads to local relative phases of two waves switching randomly between 0 and $\pi$ at each position except for a correlation point in between, which length is given by the PRBS bit duration (see Fig. 1a). Since Brillouin interaction is a phase-dependent process the acoustic wave will grow up to the steady state only in

Fifth European Workshop on Optical Fibre Sensors, edited by Leszek R. Jaroszewicz, Proc. of SPIE Vol. 8794, 87943 · @ 2013 SPIE · CCC code: 0277-786X/13/\$18 · doi: 10.1117/12.2026169 
correlation points, while for the rest of the fibre the acoustic wave amplitude will remain very small (see Fig. 1b). Thus, the change in probe power due to Brillouin gain will be given by a strong interaction at a single point (where the correlation peak is located) and a background noise coming from the rest of the fibre. Correlation points are separated by the PRBS duration, therefore a higher-order correlation point can be repositioned by a minor change in the bit duration.

(a)

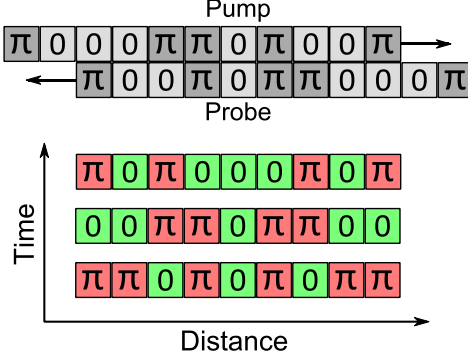

(b)

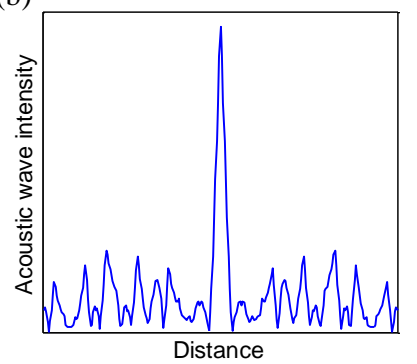

(c)

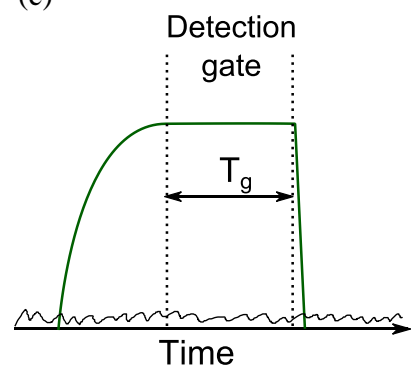

Figure 1. (a) Phase-modulated pump and probe waves and local relative phase, (b) acoustic wave intensity around the correlation point, (c) time-domain response of the correlation point and time gated detection.

In this work we have replaced the continuous pump wave by a pulse much longer than the acoustic phonon lifetime, while maintaining the pseudo-random phase modulation on pump and probe. Fig. 1c shows the expected evolution of the Brillouin gain in time-domain: once the pump pulse reaches the correlation point the probe amplification grows up until it reaches the steady state condition and quickly goes to zero after the pulse passes through. Time gating has to be used to detect just the steady-state amplification level while ignoring the rest, non-informative and noisy fibre locations. As it was mentioned before, for classical phase-correlation technique, noise comes from weak random gratings generated along the whole sensing fibre. However, for intensity-modulated pump and time-gated detection the noise level is given by the pulse duration. Therefore the SNR is improved by a factor equal to the ratio between the pulse duration and the propagation time of the fibre.

Considering the significant SNR enhancement that can be achieved with the proposed method, much longer distances can be reached. However, for longer fibres nonlinear effects should be taken into account. Actually, for the proposed method modulation instability and Raman scattering limit the pump power and therefore the SNR ${ }^{5}$, similarly to standard BOTDA sensing schemes. Four-wave mixing (FWM) can also turn significant for the phase-correlation method since the phase modulation with a PRBS spreads the spectrum of the pump and probe into numerous lines.

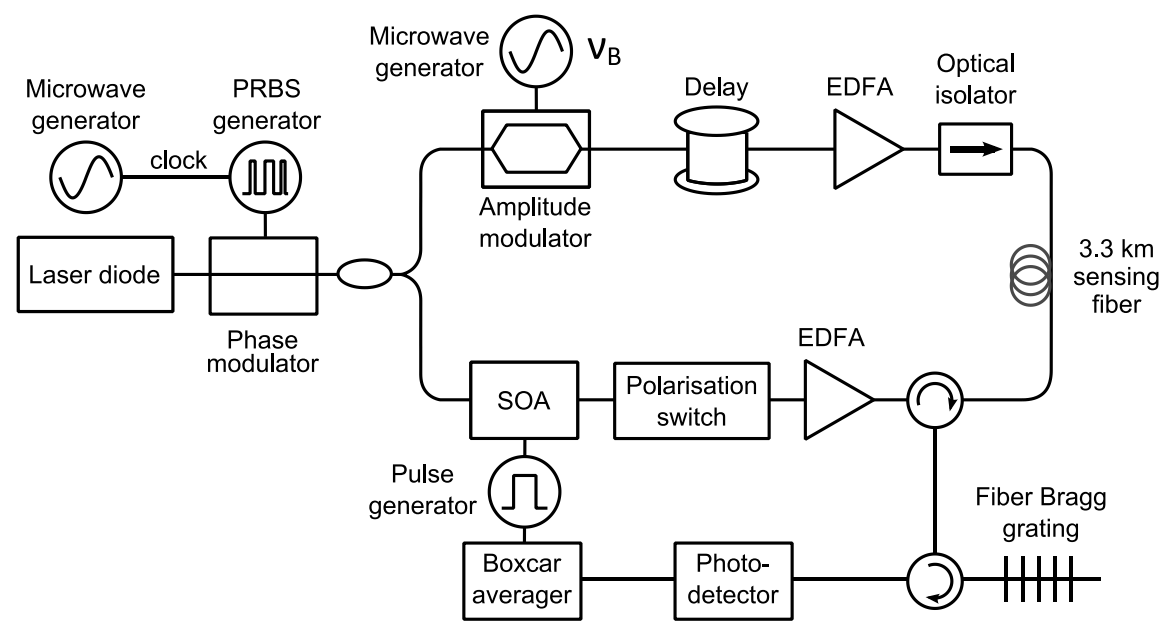

Figure 2. Experimental setup. PRBS: pseudo-random bit sequence; SOA: semiconductor optical amplifier; EDFA: erbium-doped fibre amplifier.

\section{SETUP}

The experimental setup is mostly similar to a classic BOTDA, as shows Fig. 2. The key difference is a phase modulator we place before splitting the light of a laser diode (LD) into two branches. One branch for the probe goes through an 
electro-optical modulator (EOM) driven by a microwave generator (to generate two sidebands) followed by a $10.6 \mathrm{~km}$ delaying fibre to ensure that higher-order correlation points are used for the measurement (we used correlations from the $10^{\text {th }}$ to the $19^{\text {th }}$ ). Then the probe is amplified and launched into a $3.3 \mathrm{~km}$ standard single-mode fibre. The other branch, for pump generation, contains a semiconductor optical amplifier (SOA) driven by a pulse generator, a polarization switch (in order to address both polarizations during the measurement), an EDFA and finally the sensing fibre.

An optical pulse of $\sim 100 \mathrm{~ns}$ duration has been used, corresponding to a $10 \mathrm{~m}$ interaction length region, which should lead to 330 times better SNR in comparison to continuous pump setup for $3.3 \mathrm{~km}$ sensing fibre. To give the acoustic wave time to grow to steady state condition the boxcar averager window duration was set to $T_{g}=60 \mathrm{~ns}$ (see fig. 1c). Since a low-frequency signal is measured, a $125 \mathrm{MHz}$ detector and a $15 \mathrm{MHz}$ low-pass filter have been used to reduce electrical noise. As an alternative, a much slower photodetection can be used for a further SNR improvement.

The procedure to perform a measurement at any point along the fibre is the following: the PRBS bit duration is set to a value that gives a correlation peak at the desired position and the boxcar averager window is adjusted to get the best response. After that, the microwave generator frequency is scanned over the desired Brillouin frequency range. In order to know the spectrum of the background noise, another measurement has been acquired with the same boxcar-averager window position, but with the correlation point moved away from it. However, first measurements have shown that for an intensity modulated pulse of $100 \mathrm{~ns}$ pulse, the noise level from randomly generated gratings remains below the detection limit, and therefore can be ignored during the data processing.

\section{RESULTS}

Measurements of the Brillouin frequency shift have been taken for a $3.3 \mathrm{~km}$ long fibre with a spatial resolution of $1.1 \mathrm{~cm}$ at the end of the fibre (PRBS bit duration of 110 ps). Results of this measurement can be seen on Fig. 3a (gain spectrum) and Fig. 3b (extracted Brillouin frequency shift). It is possible to see a drift in the Brillouin frequency at the end of the fibre, which could be a sign of pump depletion as in any standard BOTDA system ${ }^{6}$. In order to ascertain that this is not the case a measurement with swapped fibre ends has been carried out, and the same shape has been obtained (Fig. 3b, green line). Another proof would be the short section of the fibre at the end (loosened from the spool to make a hotspot measurement) that shows the same Brillouin frequency as the most of the fibre (Fig. 3b, inset).

(a)

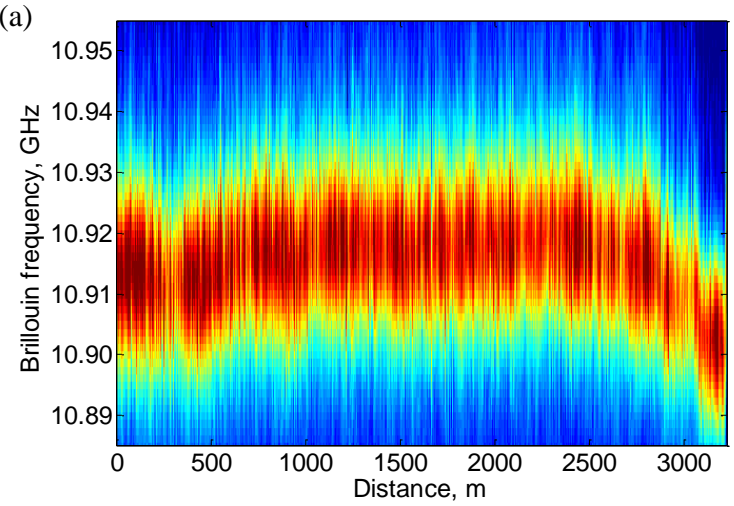

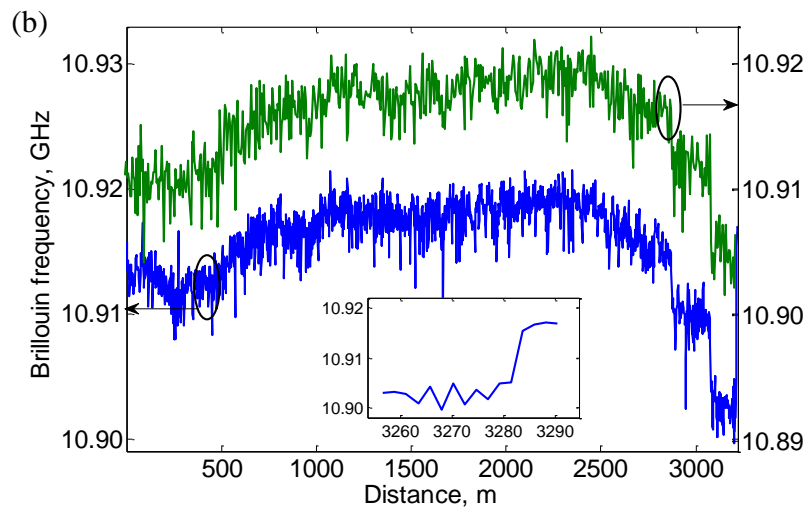

Figure 3. (a) Brillouin gain spectrum for $3.3 \mathrm{~km}$ long fibre, (b) extracted Brillouin frequency for two measurements.

No visible effect of either pump depletion or spontaneous amplified Brillouin scattering (SpABS) for the probe can be observed along the fibre. Actually, phase modulation broadens the spectrum thus preventing SpABS and short interaction length leads to negligible changes in pump power. However, modulation instability (MI) limits the power level for pump wave. In order to avoid MI dispersion shifted fibres (DSF) can be used but strong FWM has been observed on a preliminary experiment, limiting the power to only several tens of milliwatts and thus making measurements impossible.

To check the spatial resolution of our system, a $1.1 \mathrm{~mm}$ long hotspot was applied at the end of the fibre. Measurements have been performed sampling every $2 \mathrm{~mm}$. As can be seen from Fig. 4 the hotspot is clearly resolved and its measured size corresponds to the $1.1 \mathrm{~cm}$ size of the correlation point. It is possible to further improve the spatial resolution, but it leads to several problems. On one hand, the real signal decreases due to reduced interaction length, and, on the other 
hand the noise level rises as the number of random bits within the $100 \mathrm{~ns}$ pump pulse increases. In addition, as the PRBS clock approaches the Brillouin frequency (about 10-11 GHz), spectra of both probe sidebands overlap each other, making it impossible to separate them with a fibre Bragg grating.
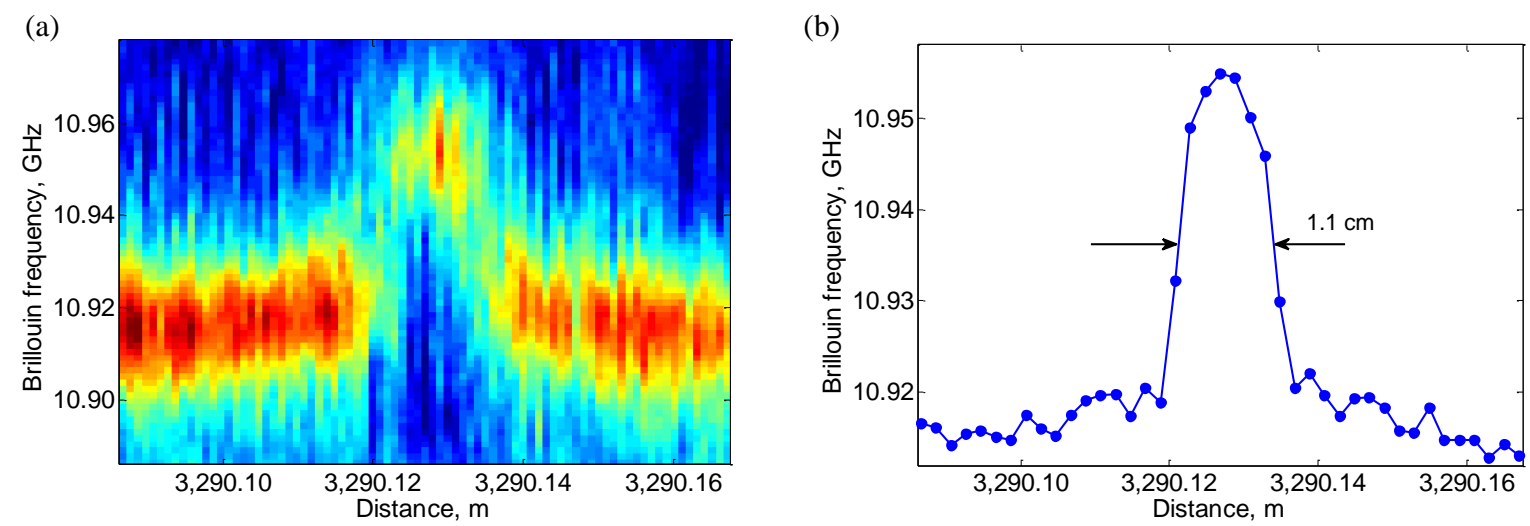

Figure 4. (a) Brillouin gain spectrum for the section of the fibre attached to a hotspot, (b) extracted Brillouin frequency.

\section{CONCLUSION}

In conclusion a distributed measurement with $1.1 \mathrm{~cm}$ spatial resolution over $3.3 \mathrm{~km}$ has been demonstrated thus making a sensor offering 300'000 resolved points. Combining high spatial resolution with long distances brings together the problems from both fields. The signal to be detected is two orders of magnitude lower than the one for BOTDA setups and the pump power is limited by modulation instability. Usually DSF can be used to avoid MI, but due to the phase modulation the pump spectrum shows multiple lines and practically four-wave mixing rapidly limits the power for DSF. These are the first results obtained with this new technique that already lead to a record number of distinct measured points. Optimisation of the pulse duration and power levels should further lead to an improved number of resolved points, with the challenging ambition to reach 1 million resolved points.

\section{REFERENCES}

[1] Foaleng, S. M., Tur, M., Beugnot, J.-C., Thevenaz, L., "High spatial and spectral resolution long-range sensing using Brillouin echoes," J. Lightwave Technol. 28 (20), 2993-3003 (2010)

[2] Dong, Y., Zhang, H., Chen, L. and Bao, X., "2 $\mathrm{cm}$ spatial-resolution and $2 \mathrm{~km}$ range Brillouin optical fiber sensor using a transient differential pulse pair,”, Appl. Opt. 51 (9), 1229-1235 (2012).

[3] Mizuno, Y., He, Z. and Hotate, K., "Measurement range enlargement in Brillouin optical correlation-domain reflectometry based on double-modulation scheme," Opt. Express 18 (6), 5926-5933 (2010)

[4] Zadok, A., Antman, Y., Primerov, N., Denisov, A., Sancho, J. and Thevenaz, L., "Random-access distributed fiber sensing," Laser Photon. Rev.6, L1-L5 (2012).

[5] Foaleng, S. and Thevenaz, L., "Impact of Raman scattering and modulation instability on the performances of Brillouin sensors," Proc. of SPIE, 7753, 77539V (2011)

[6] Thevenaz, L., Foaleng, S. and Lin, J., "Impact of pump depletion on the determination of the Brillouin gain frequency in distributed fiber sensors," Proc. of SPIE, 7753, 775322 (2011) 